\title{
Identification and characterization of microRNAs expressed in chicken skeletal muscle
}

\author{
A.P.D. Andreote ${ }^{1,2}$, M.F. Rosario ${ }^{1}$, M.C. Ledur ${ }^{3}$, E.C. Jorge ${ }^{1}$, \\ T.S. Sonstegard ${ }^{4}$, L. Matukumalli ${ }^{4}$ and L.L. Coutinho ${ }^{1}$ \\ ${ }^{1}$ Departamento de Zootecnia, Escola Superior de Agricultura "Luiz de Queiroz", \\ Universidade de São Paulo, Piracicaba, SP, Brasil \\ ${ }^{2}$ Centro de Energia Nuclear na Agricultura, Universidade de São Paulo, \\ Piracicaba, SP, Brasil \\ ${ }^{3}$ Embrapa Suínos e Aves, Concórdia, SC, Brasil \\ ${ }^{4}$ Beltsville Area Research Center, United Center Department of Agriculture, \\ Beltsville, MD, USA
}

Corresponding author: L.L. Coutinho

E-mail: 1lcoutinho@usp.br

Genet. Mol. Res. 13 (1): 1465-1479 (2014)

Received March 13, 2013

Accepted October 25, 2013

Published March 6, 2014

DOI http://dx.doi.org/10.4238/2014.March.6.5

\begin{abstract}
MicroRNAs (miRNAs, miRs) encompass a class of small non-coding RNAs that often negatively regulate gene expression. miRNAs play an essential role in skeletal muscle, determining the proper development and maintenance of this tissue. In comparison to other organs and tissues, the full set of muscle miRNAs and its expression patterns are still poorly understood. In this report, a chicken skeletal muscle miRNA library was constructed, and the expression of selected miRNAs was further characterized during muscle development in chicken lines with distinct muscling phenotypes. Clone library sequence analysis revealed 40 small RNAs with similarities to previously described chicken miRNAs, seven miRNAs that were never identified before in chicken, and some sequence clusters representing other possible novel miRNAs. Temporal expression profiles of three miRNAs associated with cell proliferation and differentiation (miR-
\end{abstract}


125b, miR-221, and miR-206) in two chicken lines (broiler and layer) revealed the differential steady-state levels of these miRs during skeletal muscle growth and suggests that miR-206 is involved in the muscling phenotype that is observed in growth-selected chicken lines.

Key words: Cell proliferation; miR-125b; miR-221; miR-206; Myogenesis

\section{INTRODUCTION}

The recent discovery of small non-coding RNAs significantly changed the considerations that must be made in evaluating the control of gene expression for any single transcript. The possibility that one unique, small RNA is capable of altering the expression of several genes in a range of different biological processes aroused our attention to the possible application of these regulatory mechanisms in different biological processes, ranging from cell differentiation to the control of disease conditions.

MicroRNAs (miRNAs) are a class of small RNAs that are crucial to the proper development and functioning of superior organisms (Berezikov et al., 2006). They can be a major determining factor in cell fate and behavior (Mukherji et al., 2011). This group of non-coding RNAs corresponds to small non-coding and evolutionarily conserved RNAs (approximately 20 nucleotides) that negatively regulate gene expression. These small RNAs recognize partial or total complementary sequences on 3' untranslated regions (3'-UTRs) of mRNAs, allowing targeted selection by the miRNA for degradation or translational repression (Filipowicz et al., 2008).

Studies concerning miRNA expression profiles have contributed to the understanding of several biological processes including tissue development and maintenance, in which cell proliferation, cell differentiation, and the balance between the two play principal roles. These molecules have been reported to play an important role in the regulation of several processes during muscular development (Cardinalli et al., 2009; Goljanek-Whysall et al., 2012). Expression of miRNAs is highly regulated spatially and temporally during development of all tissues and species studied, demonstrating the importance of these molecules in the regulation of gene expression.

MiRNAs have been identified in chicken by in silico analysis and cloning from several chicken cell lines during different developmental stages. Currently, the total number of mature chicken miRNAs identified corresponds to 996 different sequences (miRBase, release June 20, 2013). Many miRNAs have been identified in muscle, and some of these are specifically or preferentially expressed in this tissue ("myomiRs"). The regulation of miRNA expression in skeletal muscle is closely associated with essential genes for myogenesis, such as myogenic regulatory factors and fibroblast growth factors (Rosenberg et al., 2006; Sweetman et al., 2006, 2008; Goljanek-Whysall et al., 2011). Changes in miRNA expression patterns have been associated with muscular disorders such as hypertrophy and cardiac arrhythmias, atrophy, and muscular dystrophy (Caré et al., 2007; Cheng et al., 2007; Van Rooij et al., 2007). Furthermore, miRNAs have been implicated in muscling phenotypes in animals (Clop et al., 2006).

To further investigate the involvement of miRNAs in myogenesis, a chicken skeletal muscle miRNA library was constructed, and expression patterns during muscle development for selected miRNAs were further characterized in chicken lines with distinct muscling phenotypes. 


\section{MATERIAL AND METHODS}

\section{Animal sample and RNA extraction}

Two chicken lines that were developed by Embrapa Poultry Breeding Program, a broiler (TT) and a layer (CC), were used for this study. The TT line was selected for body weight, feed conversion, carcass yield, viability, fertility, hatchability, and reduced abdominal fat. The CC line was selected for egg production, egg weight, feed conversion, hatchability, sexual maturity, fertility, viability, egg quality, and reduced body weight (Rosario et al., 2009).

Pectoral muscle samples were collected during two embryonic stages, corresponding to Hamburger and Hamilton stages HH-35 (nine days of incubation, 9e) and HH-43 (17 days of incubation, 17e), and 21 days post-hatching (21a) (Hamburger and Hamilton, 1992). These stages were selected according to specific physiological characteristics of the muscle cells. At $9 \mathrm{e}$, the pectoral muscle appears newly formed. This is the first developmental stage according to Hamburger and Hamilton (1992) in which it is possible to define this muscle. Furthermore, the $9 \mathrm{e}$ stage is characterized by an intense cell proliferation rate. The $17 \mathrm{e}$ represents an intermediate phase. Because of the different animal requirements after birth (around 19 days of incubation), $17 \mathrm{e}$ is characterized by decreased cell proliferation and increased differentiation. Lastly, stage 21a corresponds to differentiated muscle cells.

Fertilized eggs from both lines were incubated in a hatchery at $37^{\circ} \mathrm{C}$ and $87 \%$ relative humidity. After hatching, chickens were kept in a heated barn until slaughter (21st day), which was performed according to international standards of animal welfare and approved by the animal ethical committee from Escola Superior de Agricultura "Luiz de Queiroz".

Samples were snap-frozen in liquid nitrogen immediately after animal slaughter. In total, 24 samples were obtained from the CC line and 32 from the TT line. Eight animals from the TT line were used for library construction, and 24 animals from each line (eight per stage) were used for quantitative reverse transcription polymerase chain reaction (RT-PCR).

Samples were processed for total RNA extraction with TRIzol (Invitrogen, USA), according to manufacturer instructions. After extraction, RNA concentration was assessed by spectrophotometry at $260 \mathrm{~nm}$, and RNA quality was assessed by the $\mathrm{OD}_{260} / \mathrm{OD}_{280} \mathrm{~nm}$ ratio and electrophoresis on $1 \%$ agarose gels $(\mathrm{w} / \mathrm{v})$.

\section{Small-RNA library construction}

Total RNA from broilers (TT) 21 days after hatching was pooled for miRNA cloning using the miRCat small RNA cloning kit (IDT DNA Technologies, USA) following manufacturer instructions with few modifications. Briefly, $500 \mu \mathrm{g}$ pooled total RNA was size fractioned using $15 \%$ denaturing polyacrylamide gel electrophoresis (PAGE). A portion of about 20 nucleotide size was sliced from the gel, and RNA was isolated and eluted by FlashPAGE electrophoresis (Ambion, USA). Next, 3' linkers (5' phosphorylated) were ligated to the small RNA fraction in the absence of ATP. The resulting sample was subjected to a new fractionation using $10 \%$ PAGE, and $5^{\prime}$ linkers were ligated in the presence of ATP. Then, the mix was reverse transcribed using a primer complementary to the $3^{\prime}$ linker sequence and PCR-amplified using primers for both linkers. Amplified products were cloned using the pGEM-T Easy Vector cloning kit (Promega, USA) following the manufacturer protocol. Individual colonies of host bacterium Escherichia coli DH5- $\alpha$ 
were picked, and the plasmid DNA was extracted. The sequencing reaction $(10 \mu \mathrm{L})$ contained $1 \mathrm{X}$ sequencing buffer (Applied Biosystems, USA), $2 \mu \mathrm{L}$ Big Dye Terminator mix (Applied Biosystems), and $5 \mu \mathrm{M}$ M13 primer. Ready-to-sequence DNA templates were isopropanol-cleaned and resuspended in $3 \mu \mathrm{L}$ HiDi formamide (Applied Biosystems). Sequencing was performed with the DYEnamic ET DyeTerminator Sequencing Kit (GE Healthcare Bio-Science AB, Sweden) and the ABI 3100 Automated DNA Sequencer (Applied Biosystems).

\section{Sequence analyses}

All chromatograms were analyzed with Phred (Ewing et al., 1998) and screened for vector and linker sequences. Clones with inserts between 17 and 30 bases and a minimum base quality of 20 for all bases were further analyzed. Distinct clone sequences were clustered and assembled to obtain the longest sequence, along with member counts within the cluster. Some of the clusters were further collapsed by manual intervention that allowed for single base mismatch corrections, especially toward the ends of cloned sequences.

The miRNAs were identified and named based on sequence homology to published miRNAs in the miRBase (http://microrna.sanger.ac.uk) and NCBI (www.ncbi.nlm.nih.gov) databases. No match sequences were screened against the tRNA (http://lowelab.ucsc.edu/ GtRNAdb/Hsapi/Hsapi-summary.html) and snoRNAs (http://www-snorna.biotoul.fr/browse. php) databases to remove sequences that could interfere with the identification of novel chicken miRNAs. For the non-identified sequences, genomic regions containing inserts with 100 nucleotide flanks were retrieved from Ensembl release 52 Gallus gallus genome annotation version WASHUC2 (http://www.ensembl.org), and a sliding window of 100 nucleotide was used to calculate RNA secondary structures by RNAfold (Zucker, 2003).

\section{Quantitative RT-PCR}

Two lines (TT and CC) at three developmental stages (9e, 17e, and 21a) were compared in the quantitative miRNA expression analysis. We analyzed the expression patterns of three miRNAs according to miRBase (http://www.mirbase.org/): miR-125b (MI0001175), miR-221 (MI0001178), and miR-206 (MI0001207). We applied 5S rRNA as a reference. The quantification of these targets was conducted in a total of 48 animals (eight of each stage per line). Quantitative RT-PCR was conducted using the miRVana qRT-PCR miRNA Detection kit (Applied Biosystems) on a LightCycler system (Roche Diagnostics, Mannheim, Germany). For the RT reaction, $25 \mathrm{ng}$ total RNA was used. The RT reaction contained $2 \mu \mathrm{L} 5 \mathrm{X}$ RT buffer, $1 \mu \mathrm{L} 1 \mathrm{X}$ RT primer, and $0.4 \mu \mathrm{L}$ enzyme mix. Reaction conditions were $30 \mathrm{~min}$ at $37^{\circ} \mathrm{C}$ and $10 \mathrm{~min}$ at $95^{\circ} \mathrm{C}$. The PCR comprised the entire RT product, $5 \mu \mathrm{L} 5 \mathrm{X}$ PCR buffer, $0.5 \mu \mathrm{L}$ primers, and $3 \mathrm{U}$ SuperTaq DNA polymerase (Ambion). Reaction conditions were $95^{\circ} \mathrm{C}$ for $3 \mathrm{~min}$, and 40 cycles at $95^{\circ} \mathrm{C}$ for $15 \mathrm{~s}$ and $60^{\circ} \mathrm{C}$ for $30 \mathrm{~s}$. The specificity of the amplification of each transcript was confirmed by melting curve analysis that was performed at temperatures ranging from 60 to $96^{\circ} \mathrm{C}$, where unique peaks were observed.

\section{Statistical analysis}

Samples from each animal were normalized using the expression: $\triangle \mathrm{CT}=\mathrm{CT}_{\text {target }}$ $\mathrm{CT}_{\text {reference }}$, in which target genes were miR-125b, miR-221, and miR-206, and the reference 
gene was $5 \mathrm{~S}$ rRNA. Higher $\triangle \mathrm{CT}$ values correspond to lower gene expression levels. The experiment had a completely random design in a $2 \times 3$ factorial scheme ( 2 lines and 3 stages) with each animal being considered an experimental unit $(\mathrm{N}=8$ per treatment). Analysis of variance (ANOVA) $(\mathrm{df}=42)$ was employed to find effects for lines, stages, and the lines $\mathrm{x}$ stages interaction. When some significant effect was found, means \pm standard error (SE) of $\triangle \mathrm{CT}$ were compared by least squares $(\mathrm{P}<0.05)$ using the PROC GLM program in SAS. The assumptions of ANOVA were verified by SASLAB of SAS; specifically in the case of miR-206, a base-10 log scale was necessary to use these assumptions for data processing (SAS, 2007).

\section{RESULTS}

\section{Identification of muscle miRNAs expressed}

A chicken skeletal muscle miRNA cDNA library was constructed from size-fractioned pectoral muscle RNA (15-30 bases). Basic local alignment search tool analysis of 361 high quality small RNA-derived sequences against miRBase (Griffiths-Jones et al., 2006; http:// microrna.sanger.ac.uk) resulted in the identification of 40 sequence clusters similar to chicken miRNAs, seven sequence clusters with high or perfect homology to miRNAs from other species that had not been previously identified in chicken, and three clusters that were not represented in miRBase (Figures 1 and 2).

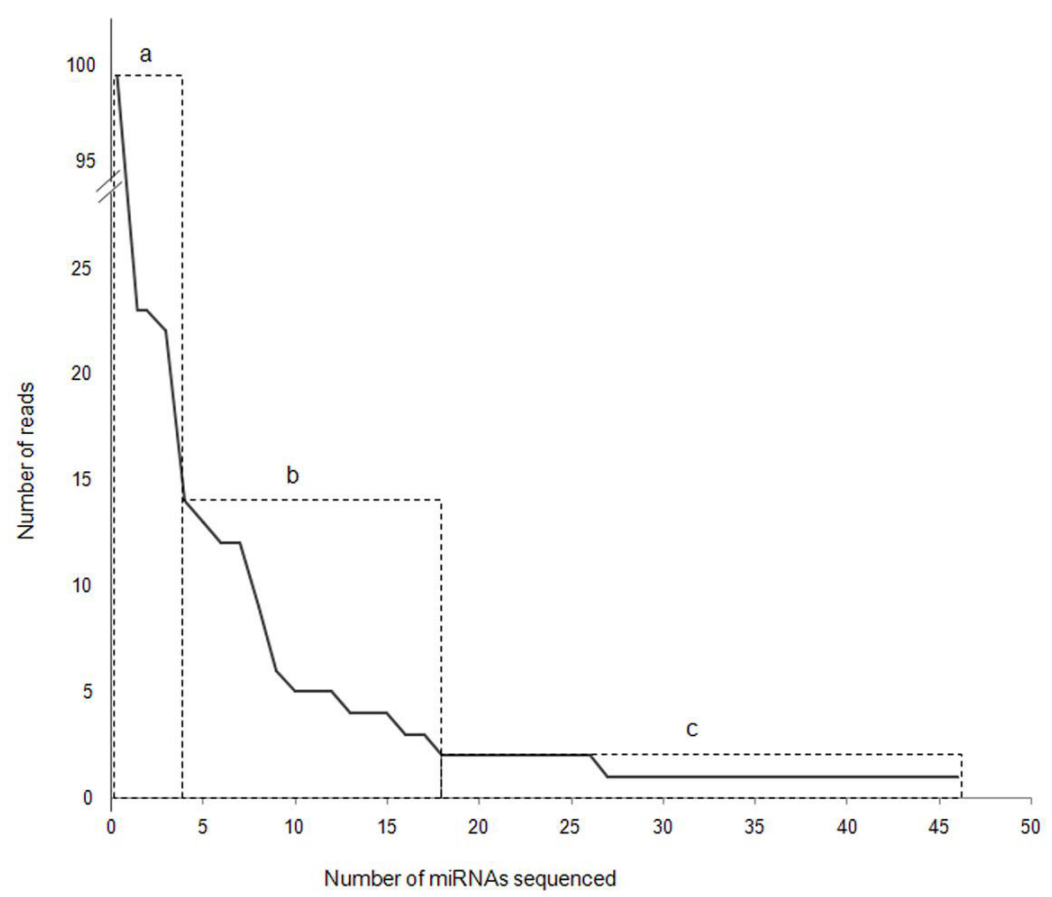

Figure 1. Abundance of miRNAs identified in the library from skeletal muscle at 21 day post-hatch chicken. miRNAs presented: high (A), medium (B), and minimum (C) abundance. 


\begin{tabular}{|c|c|}
\hline A & $1 \ldots \ldots 10 \ldots 20$ \\
\hline$g g a-m i R-27 a$ & UUCACAGUGGCUAAGUU \\
\hline hsa-miR-27a & UUCACAGUGGCUAAGUU \\
\hline$m m u-m i R-27 a$ & UUCACAGUGGCUAAGUU \\
\hline oan-miR-27a & UUCACAGUGGCUAAGUU \\
\hline B & $1 \ldots \ldots . \ldots 20 \ldots 30$ \\
\hline gga-miR-214* & --CCUGUCUACACUUGCUGUGC \\
\hline hsa-miR-214* & UGCCUGUCUACACUUGCUGUGC \\
\hline oan-miR-214* & UGCCUGUCUACACUUGCUGU-- \\
\hline $\mathrm{C}$ & $1 \ldots \ldots 10 \ldots 20 \ldots 30$ \\
\hline gga-miR-425 & AAUGACACGAUCACUCCŪGCUGAGC \\
\hline hsa-miR-425 & AAUGACACGAUCACUCCCGUUGA-- \\
\hline$m m u-m i R-425$ & AAUGACACGAUCACUCCCGUUGA-- \\
\hline oan-miR-425 & AAUGACACGAUCACUCCCGUUGA-- \\
\hline $\mathrm{D}$ & $1 \ldots \ldots 10 \ldots \ldots 20 \ldots 30$ \\
\hline gga-miR-363 & -ACAGAUGGAUACCGUGCAAUU \\
\hline hsa-mi R-363 & UACAGAUGGAUACCGUGCAAUU \\
\hline $\mathrm{mmu}-\mathrm{mi} \mathrm{R}-363$ & UACAGAUGGAUACCGUGCAAUU \\
\hline oan-miR-363 & -ACAGAUGGAUACCGUGCAAUU \\
\hline $\mathrm{E}$ & $1 \ldots \ldots 10 \ldots 20 \ldots 30$ \\
\hline gga-miR-26b* & CCUGUUCUĀGGUUACUUGGCU- \\
\hline hsa-miR-26b* & CCUGUUCUCCAUUACUUGGCUC \\
\hline$m m u-m i R-26 b^{*}$ & CCUGUUCUCCAUUACUUGGCUC \\
\hline oan-miR-26* & CCUGUCCUCGGUUACUUGUCUC \\
\hline $\mathbf{F}$ & $1 \ldots \ldots 10 \ldots 20 \ldots 30$ \\
\hline$g g a-m i R-23 a$ & AUCACAUUGCCG̈GGGAUUUCCAA \\
\hline hsa-miR-23a & AUCACAUUGCCAGGGAUUUCC-- \\
\hline$m m u-m i R-23 a$ & AUCACAUUGCCAGGGAUUUCC-- \\
\hline oan-miR-23a & AUCACAUUGCCAGGGAUUUCCA- \\
\hline G & $1 \ldots \ldots 10 \ldots \ldots 20 \ldots 30$ \\
\hline gga-miR-1335 & GCCGGCUGAGGUGGGĀUCCCGGGGCGG \\
\hline oan-miR-1335 & GUGGGCUCCCU------ \\
\hline
\end{tabular}

Figure 2. Alignment of the seven cloned chicken miRNAs (A to $\mathbf{G})$ with highly homologous miRNAs from other species.

\section{Expression profile of miR-125b, miR-221, and miR-206}

miRNAs miR-125b, miR-206, and miR-221 were further investigated in chicken lines with different muscling potential. These miRNAs were selected for expression analysis because of the following features: i) they were found in the library, and ii) all of these miRNAs 
are involved in the control of cell proliferation and cell differentiation, which are key events for myogenesis. The expression of miR-125b (Figure 3A), miR-221 (Figure 3B), and miR-206 (Figure 3C) was determined in three developmental stages (9e, 17e, and 21a) in two chicken lines (TT and CC). Ct values for the control gene, 5S rRNA, ranged from 10.9 to 11.5 in the 48 samples analyzed and did not differ significantly between stages or lines.

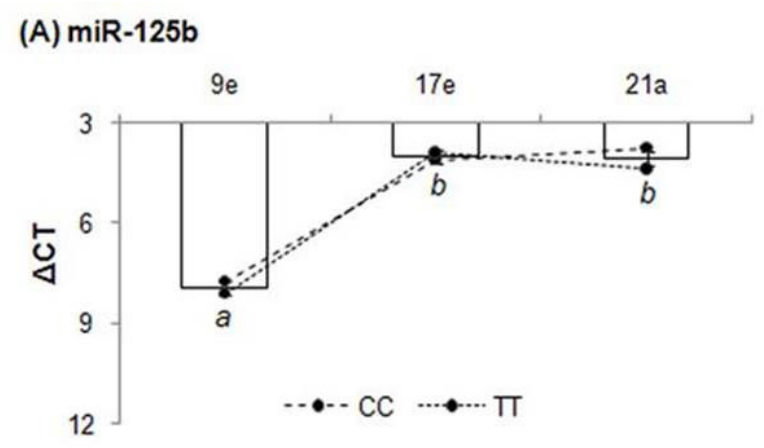

(B) $m i R-221$

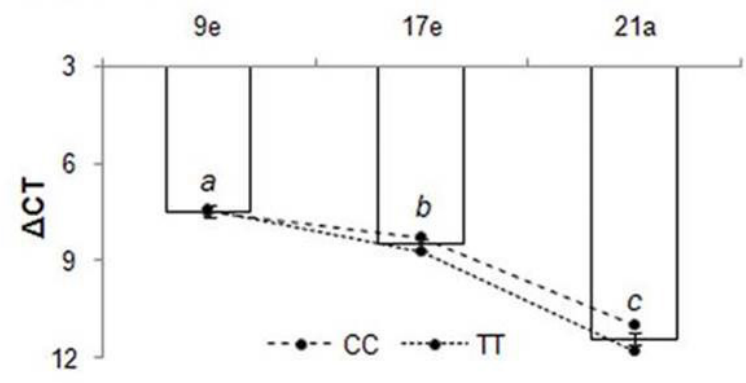

(C) $\mathrm{miR}-206$

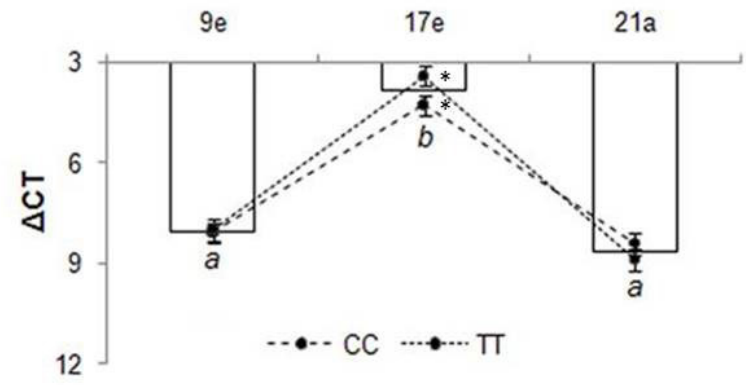

Figure 3. Expression patterns of miR-125b (A), miR-221 (B), and miR-206 (C) during chicken muscle development by quantitative RT-PCR. Stages analyzed include embryos at 9 and 17 days of incubation and adults at 21 days posthatching. The means of the normalized gene expression values for each stage were calculated and expressed as $\Delta \mathrm{CT}$ $(>\Delta \mathrm{CT} \rightarrow<$ gene expression). Error bars indicate standard errors. Different letters indicate significance between stages and asterisk between lines $(\mathrm{P}<0.05)$. 
No interaction was evident, but each miRNA showed a particular expression profile during muscle development, and the overall pattern of all miRNAs shows similar expression between the lines, with differences in distinct stages of development (Figure 3). At stage 9e, miR-125b, miR-221, and miR-206 had similar expression levels. miR-125b and miR-206 had higher expression at stage $17 \mathrm{e}$ than $9 \mathrm{e}$, while miR-221 had lower expression. At stage $21 \mathrm{a}, \mathrm{miR}-125 \mathrm{~b}$ maintained the same expression level that was observed at $17 \mathrm{e}$, and miR-221 expression was further reduced, while miR-206 returned to the expression level that was observed at $9 \mathrm{e}$. When the comparison was made between lines, only miR-206 presented a higher expression level in the broiler (TT) line at stage 17e (Figure 3).

\section{DISCUSSION}

\section{Identification of miRNAs expressed in adult chicken skeletal muscle}

Data concerning miRNA expression in several tissues and cell lines have helped to identify miRNAs that can control several processes by repressing specific genes. In this study, we analyzed pectoral muscle of chick embryos, and we identified 40 sequence clusters that were similar to chicken miRNAs, seven sequence clusters with high or perfect homology to miRNAs from other species that had not been previously identified in chicken, and three clusters that were not represented in mirBase.

Among the miRNAs identified, miR-133 was the most abundant (98 reads, 27\%). miR-1, miR-126*, and miR-1335 were also highly represented (about 20 reads each, 5\%) (Figure 1a). A group of 14 miRNAs showed medium coverage (3 to 14 reads each) (Figure 1b). Moreover, 9 miRNAs had two reads each, and 20 miRNAs had minimal coverage, with one read each (Figure 1c).

Sequenced miRNAs similar to other species but not previously identified in chicken were annotated as gga-miR-27a, gga-miR-214*, gga-miR-425, gga-miR-363, gga-miR-26b*, gga-miR-23a, and gga-miR-1335 (Figure 2 and Table 1). Of these miRNAs, gga-miR-1335 and gga-miR-363 could be aligned to the chicken genome assembly. The other five sequences could not be located. The inability to localize some sequences was reported in another study that associated this absence with the incomplete sequence coverage of the chicken genome (Shao et al., 2008; Li et al., 2012).

gga-miR-27a was identical to human, mouse, and platypus miR-27a, suggesting the high conservation of this miRNA among vertebrates. gga-miR-214*, gga-miR-425, ggamiR-363, gga-miR-26b*, and gga-miR-23a differed between the species analyzed by at least one nucleotide. This difference might have some impact on target recognition and post-transcriptional regulation. miR-425 is 25 nucleotides long in chicken, but it is 23 nucleotides long in all other species analyzed. The sequence of gga-miR-363 was identical to that of the platypus, and it differed in one nucleotide in other vertebrates.

In addition to the differences at the $3^{\prime}$ end of the sequences, miR-26b* and miR-23a showed a single base substitution: $\mathrm{C}$ to $\mathrm{A}$ at position 9 and $\mathrm{A}$ to $\mathrm{G}$ at position 12 , respectively. These mismatches may not have profound influences on target recognition and mRNA inhibition because they are not in the seed region (the first 6-8 nucleotides from the $5^{\prime}$ end), but it is likely that they are responsible for fine-tuning the expression of their targets (Filipowicz et al., 2008). 


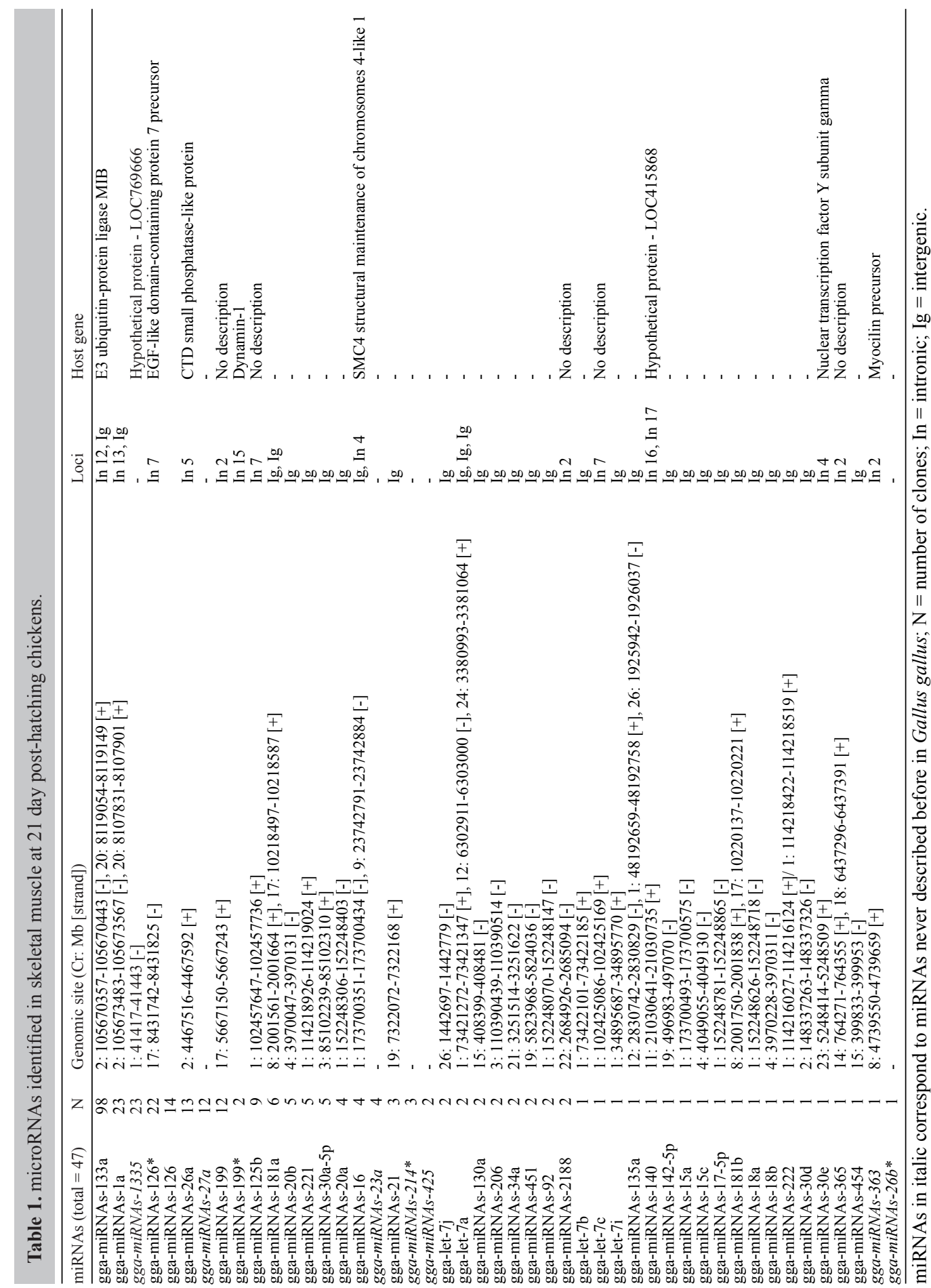


The identification of putative novel miRNAs was based on the following criteria. First, the mature miRNA must be expressed as a distinct transcript with an approximate length of 25 nucleotides. Second, the mature miRNA must originate from a precursor with a characteristic secondary structure, such as a hairpin or fold-back, that does not contain large internal loops or bulges. Third, an optional but commonly used criterion is that mature miRNA sequences should be conserved in different species (Berezikov et al., 2006). We had three clusters (five reads) of putative novel chicken miRNAs that were identified in the library, thus fulfilling the first criterion. According to the mFold software (RNAfold), two of these sequences were able to produce hairpin miRNA precursors (Figure 4A and 4B). In addition, all clusters were conserved in at least one additional species. Concerning the similarity parameter among sequences, we found that these three sequences could be considered novel miRNAs because of their similarity to families already described (Table 2).

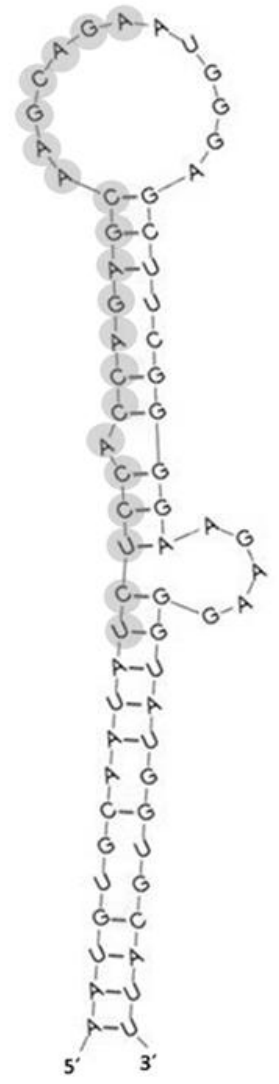

A

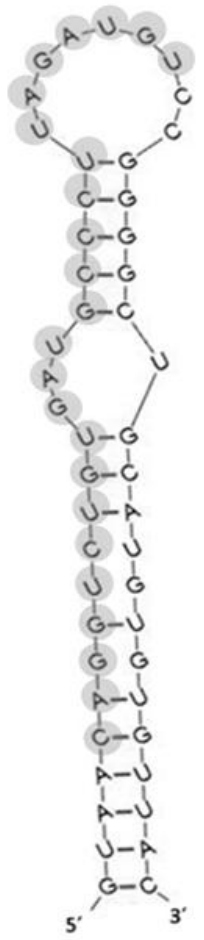

B

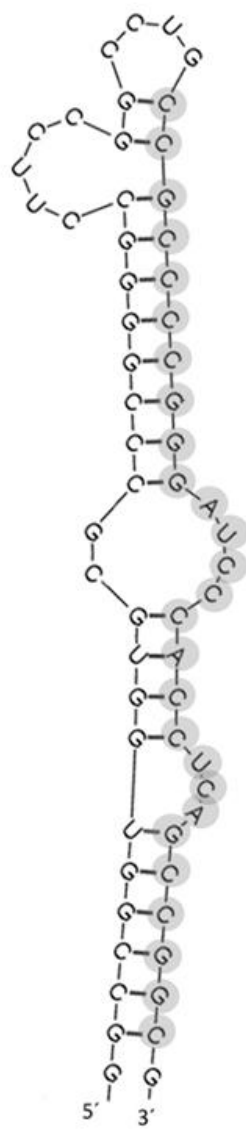

$\mathrm{C}$

Figure 4. Predicted secondary structures of the putative novel chicken miRNA precursors presented in Table 2 (A-B) and gga-miR-1335 (C). Chicken genomic sequences upstream and downstream of the novel miRNAs were folded with the RNAfold program. Gray areas represent the cloned miRNA. 


Table 2. Putative novel miRNAs cloned from chicken skeletal muscle.
\begin{tabular}{lcccccccc}
\hline miRNAs & N & miRNA family & Gga & Start & End & Conservation & Structure & dG \\
\hline TCTCCACCAGAGCAAGCAGAA & 2 & 1306 & 2 & 16283207 & 16283187 & D, H, M, O, X & A & -23.0 \\
CAGGTCTGTGATGCCCTTAGATGT & 2 & 148 & 1 & 15771 & 15794 & X, Z & B & -35.6 \\
GTTAAAAAGCTCGTAGTTGGATC & 1 & 4014 & 1 & $16589 / 2831712$ & $16611 / 2831734$ & O, X, Z & - & - \\
\hline
\end{tabular}

$\mathrm{N}=$ number of reads; Gga = chromosome assignments on Gallus gallus genome build 3.0; Start = position on Gga where miRNA sequence starts; End = position on Gga where miRNA sequence ends; Conservation = conservation of the miRNA in H (human), D (dog), M (mouse), Z (zebrafish), X (Xenopus), and O (opossum) genomes by BLAT $(\mathrm{UCSC})$; Structure $=$ the folding structure of the miRNA represented in Figure $4 ; \mathrm{dG}=$ free energy of the miRNA structure as calculated by RNAFold.

A cluster containing 98 sequences was located in a hypothetical protein (LOC769666) in chromosome 1 of the chicken genome. This sequence cluster was highly similar to oanmiR-1335, a miRNA only previously described in platypus. The miR-1335 sequence in platypus is 18 nucleotides long, whereas the sequence identified in chicken (gga-miR-1335) is 27 bases long. Additionally, gga-miR-1335 showed a double base substitution, $\mathrm{C}$ to A at position 16 and $\mathrm{U}$ to $\mathrm{G}$ at position 21. According to UCSC genome browser (http://genome.ucsc. edu/cgi-bin/hgBlat?command=start), this sequence is poorly conserved among vertebrates; however, according to RNAfold (Zuker, 2003), the chicken sequence is able to produce a hairpin miRNA precursor (Figure 4C). The similarities between that sequence cluster and oan-miR-1335, in addition to the possibility of having a hairpin precursor, suggest that this sequence might be a chicken miR-1335. Also, this sequence must have an important role in skeletal muscle because of its high abundance in the library. Additional functional assessments must be made to explore the role of this molecule in skeletal muscle.

An interesting observation from the sequencing survey was that some putative novel miRNAs found in chicken muscle are only conserved in non-mammalian species like opossums, zebrafish, and Xenopus, which suggests the conservation of these molecules in older species. The conservation and divergence of miRNAs can be an indicator of which kind of genetic information is preserved between related species (Lee et al., 2007).

\section{Expression profile of miR-125b, miR-221, and miR-206}

Skeletal muscle development in chickens and other vertebrates occurs in specific phases. Cells from the mesoderm become committed to the myogenic lineage and turn into myoblasts. After that, myoblast proliferation remains until specific signals for differentiation are received and recognized by these cells. Then, myoblasts develop into differentiated cells, which are submitted to a fusion process to form myotubes. Later, these myotubes mature into muscle fibers. This intricate program requires the action of many genes, which are expressed with temporal and spatial coordinate specificity (Mok and Sweetman, 2011). One major mechanism determining myogenesis is the balance of cell proliferation and differentiation. The control of cell proliferation and differentiation requires the coordinated action of several genes, which must be induced or inhibited at a specific time and site. The precise control of this balance determines proper muscular development, function, and regeneration capacity. This balance of control is accomplished by numerous molecules, among which the miRNAs play an important role (Goljanek-Whysal et al., 2012). Therefore, the characterization of miRNA 
expression profiles is important to understand the mechanisms controlling skeletal muscle development and maintenance.

Three miRNAs that were found in our library and were known to be involved in the control of genes associated with cell proliferation and differentiation, miR-125b, miR221 , and miR-206, were characterized by determining their expression profile and possible involvement in muscle development.

miR-125b is consistently expressed in many tissues and specific development stages. It is a critical player in regulating cell proliferation in chick embryos with a higher level of tissue-specific expression in many different embryonic structures (Hicks et al., 2008, 2009). Concerning function, Lee et al. (2005) demonstrated that when miR-125 is blocked from functioning by 2 '-O-methyl antisense oligonucleotides, there is a decrease in the proliferation of various cells types. The role of miR-125b in myogenesis modulation was elucidated recently. A study in cultured cells and muscle regeneration of mice showed a decline in miR-125b levels during myogenesis progression (Ge et al., 2011). These authors also identified insulin-like growth factor 2, a positive regulator of muscle differentiation, as a direct target of miR-125b. These findings indicate that miR-125b acts as a negative regulator of muscle cell differentiation. In addition, Hicks et al. (2008) showed that miR-125b is highly expressed in chick embryos at stage $20 \mathrm{e}$. In our study, the lowest miR-125b expression was observed at embryonic development stage $9 \mathrm{e}$, which was followed by greater expression at stage 17e and 21a (Figure $3 \mathrm{~A})$. Because skeletal muscle contains differentiated muscle fibers and proliferating satellite cells, cell type-specific expression studies need to be conducted to fully elucidate the action of miR-125b. In a different cellular context, miR-125b could act as a negative modulator of cell proliferation because it has been described as a tumor suppressor (Visone et al., 2007). miR$125 \mathrm{~b}$ expression is induced during osteoblastic differentiation, and it is higher in differentiated cells than in undifferentiated cells (Mizuno et al., 2008). These apparently antagonistic roles of miR-125b in modulating cell proliferation and differentiation illustrate the complexity of miRNA actions and highlight the need for more detailed studies on possible regulatory pathways of these molecules in each species, tissue, and development stage.

We also examined miR-221, an indirect inducer of cell proliferation. Studies have shown high miR-221 expression in many types of cancer (le Sage et al., 2007; Visone et al., 2007). In myogenic mouse cells, miR-221 is strongly downregulated upon differentiation, and levels of the cell cycle inhibitor cyclin-dependent kinase inhibitor (p27) are inversely correlated with miR-221 (Cardinali et al., 2009). These authors also showed that miR-221 can act during early and late stages of myogenesis, playing a fundamental role in the progression from myoblasts to myocytes and in achieving a fully differentiated phenotype. As shown in Figure 3B, miR-221 expression gradually decreased over the period analyzed. Our results are in agreement with the literature and suggest that miR-221 might be involved in decreased proliferation during the progression of the muscle development process in chickens.

miR-206 is highly expressed during cardiac and skeletal muscle cell differentiation (Lagos-Quintana et al., 2002; Zhao et al., 2005). Several studies indicate that miR-206 plays an important role in the growth and development of skeletal muscle. One predicted target of miR-206 is PolA1, which is the largest subunit of DNA polymerase $\alpha$ (Kim et al., 2006), inhibiting PolA1 results in decreased proliferation and a transition from proliferation to differentiation. Other predicted targets are HDAC4 (Chen et al., 2006), gap junction protein a1 (also known as connexin43; Anderson et al., 2006), calmodulin, and MEF2A (Ikeda et al., 2009). 
Satellite cells, which regulate the switch from proliferation to differentiation by controlling PAX7 (Chen et al., 2010) and PAX3 (Hirai et al., 2010; Goljanek-Whysall et al., 2011), have also been shown to express miR-206. Furthermore, miR-206 may modulate the proliferation and apoptosis of neural cells by regulating the expression of the OTX2 gene (Wang et al., 2012). In our study, we found that miR-206 expression is increased between stages $9 \mathrm{e}$ and $17 \mathrm{e}$; this is followed by a significant reduction at stage $17 \mathrm{e}$. This suggests that miR-206 acts at the onset of the differentiation phase but does not have a role in maintaining the differentiated stage.

The relative expression between the broiler and layer lines was compared for all three miRNAs, of which only miR-206 had differential expression. miR-206 was differentially expressed between broiler (TT) and layer (CC) lines, where TT presented the highest expression level at 17e (Figure 3C). The TT and CC lines have different growth and muscling potentials. At 41 days of age, TT chickens are about five-fold heavier in body weight and seven-fold heavier in pectoral muscle weight than CC individuals (Ledur et al., 2000). The differential expression of miR-206 between these lines at stage 17e suggests that this molecule could have a role in determining the muscling phenotype divergences that are observed in these lines. Because miR-206 appears to be involved in the transition from proliferation to differentiation, the higher expression of miR-206 in the TT line could indicate that there is a large population of myoblasts that differentiate into myocytes at stage $17 \mathrm{e}$, and this could help explain the larger pectoral muscle that is observed in this line.

The cloning and sequencing of mature miRNAs from chicken muscle allowed the identification of novel chicken miRNAs and the determination of their relative abundance. Expression profile studies of selected miRNAs at different developmental stages in lines with distinct muscling phenotypes contribute to our understanding of how miRNAs contribute to muscle development in growth-selected chicken lines.

\section{ACKNOWLEDGMENTS}

The authors would like to thank Francisco and Fernando D. Andreote for reviewing this manuscript. Ana Paula D. Andreote and Millor F. do Rosário received grants from FAPESP and CNPq. Luiz L. Coutinho and Millor F. Rosário are recipients of CNPq research productivity scholarships.

\section{REFERENCES}

Anderson C, Catoe H and Werner R (2006). MIR-206 regulates connexin43 expression during skeletal muscle development. Nucleic Acids Res. 34: 5863-5871.

Berezikov E, Cuppen E and Plasterk RH (2006). Approaches to microRNA discovery. Nat. Genet. 38 Suppl: S2-S7.

Cardinali B, Castellani L, Fasanaro P, Basso A, et al. (2009). Microrna-221 and microrna-222 modulate differentiation and maturation of skeletal muscle cells. PLoS One 4: e7607.

Caré A, Catalucci D, Felicetti F, Bonci D, et al. (2007). MicroRNA-133 controls cardiac hypertrophy. Nat. Med. 13: 613618.

Chen JF, Mandel EM, Thomson JM, Wu Q, et al. (2006). The role of microRNA-1 and microRNA-133 in skeletal muscle proliferation and differentiation. Nat. Genet. 38: 228-233.

Cheng Y, Ji R, Yue J, Yang J, et al. (2007). MicroRNAs are aberrantly expressed in hypertrophic heart: do they play a role in cardiac hypertrophy? Am. J. Pathol. 170: 1831-1840.

Chen JF, Tao Y, Li J, Deng Z, et al. (2010). microRNA-1 and microRNA-206 regulate skeletal muscle satellite cell proliferation and differentiation by repressing Pax7. J. Cell Biol. 190: 867-879. 
Clop A, Marcq F, Takeda H, Pirottin D, et al. (2006). A mutation creating a potential illegitimate microRNA target site in the myostatin gene affects muscularity in sheep. Nat. Genet. 38: 813-818.

Ewing B, Hillier L, Wendl MC and Green P (1998). Base-calling of automated sequencer traces using phred. I. Accuracy assessment. Genome Res. 8: 175-185.

Filipowicz W, Bhattacharyya SN and Sonenberg N (2008). Mechanisms of post-transcriptional regulation by microRNAs: are the answers in sight? Nat. Rev. Genet. 9: 102-114.

Ge Y, Sun Y and Chen J (2011). IGF-II is regulated by microRNA-125b in skeletal myogenesis. J. Cell Biol. 192: 69-81.

Goljanek-Whysall K, Sweetman D, Abu-Elmagd M, Chapnik E, et al. (2011). MicroRNA regulation of the paired-box transcription factor Pax 3 confers robustness to developmental timing of myogenesis. Proc. Natl. Acad. Sci. U. S. A. 108: 11936-11941.

Goljanek-Whysall K, Sweetman D and Münsterberg AE (2012). microRNAs in skeletal muscle differentiation and disease. Clin. Sci. 123: 611-625.

Griffiths-Jones S, Grocock RJ, van Dongen S, Bateman A, et al. (2006). miRBase: microRNA sequences, targets and gene nomenclature. Nucleic Acids Res. 34: D140-D144.

Hamburger V and Hamilton HL (1992). A series of normal stages in the development of the chick embryo. 1951. Dev. Dyn. 195: 231-272.

Hicks JA, Tembhurne P and Liu HC (2008). MicroRNA expression in chicken embryos. Poult. Sci. 87: 2335-2343.

Hicks JA, Tembhurne PA and Liu HC (2009). Identification of microRNA in the developing chick immune organs. Immunogenetics 61: 231-240.

Hirai H, Verma M, Watanabe S, Tastad C, et al. (2010). MyoD regulates apoptosis of myoblasts through microRNAmediated down-regulation of Pax3. J. Cell Biol. 191: 347-365.

Ikeda S, He A, Kong SW, Lu J, et al. (2009). MicroRNA-1 negatively regulates expression of the hypertrophy-associated calmodulin and Mef2a genes. Mol. Cell Biol. 29: 2193-2204.

Kim HK, Lee YS, Sivaprasad U, Malhotra A, et al. (2006). Muscle-specific microRNA miR-206 promotes muscle differentiation. J. Cell Biol. 174: 677-687.

Lagos-Quintana M, Rauhut R, Yalcin A, Meyer J, et al. (2002). Identification of tissue-specific microRNAs from mouse. Curr. Biol. 12: 735-739.

le Sage C, Nagel R, Egan DA, Schrier M, et al. (2007). Regulation of the p27(Kip1) tumor suppressor by miR-221 and miR-222 promotes cancer cell proliferation. EMBO J. 26: 3699-3708.

Ledur MC, Zanella EL, Schmidt GS and Jaenisch FRF (2010). Divergence of Strains and Strain Crosses used to Develop New Reference Populations for QTL Studies in Poultry. Abstracts of 21st World's Poultry Congress, Montreal.

Lee YS, Kim HK, Chung S, Kim KS, et al. (2005). Depletion of human micro-RNA miR-125b reveals that it is critical for the proliferation of differentiated cells but not for the down-regulation of putative targets during differentiation. J. Biol. Chem. 280: 16635-16641.

Lee CT, Risom T and Strauss WM (2007). Evolutionary conservation of microRNA regulatory circuits: an examination of microRNA gene complexity and conserved microRNA-target interactions through metazoan phylogeny. DNA Cell Biol. 26: 209-218.

Li T, Wang S, Wu R, Zhou X, et al. (2012). Identification of long non-protein coding RNAs in chicken skeletal muscle using next generation sequencing. Genomics 99: 292-298.

Mizuno Y, Yagi K, Tokuzawa Y, Kanesaki-Yatsuka Y, et al. (2008). miR-125b inhibits osteoblastic differentiation by down-regulation of cell proliferation. Biochem. Biophys. Res. Commun. 368: 267-272.

Mok GF and Sweetman D (2011). Many routes to the same destination: lessons from skeletal muscle development. Reproduction 141: 301-312.

Mukherji S, Ebert MS, Zheng GX, Tsang JS, et al. (2011). MicroRNAs can generate thresholds in target gene expression. Nat. Genet. 43: 854-859.

Rosario MF, Ledur MC, Moura ASAMT, Coutinho LL, et al. (2009). Genotypic characterization of microsatellite markers

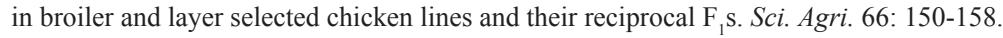

Rosenberg MI, Georges SA, Asawachaicharn A, Analau E, et al. (2006). MyoD inhibits Fstl1 and Utrn expression by inducing transcription of miR-206. J. Cell Biol. 175: 77-85.

SAS (2007). SAS/STAT User's Guide. Version 9.13, SAS Institute Inc, Cary.

Shao P, Zhou H, Xiao ZD, He JH, et al. (2008). Identification of novel chicken microRNAs and analysis of their genomic organization. Gene 418: 34-40.

Sweetman D, Rathjen T, Jefferson M, Wheeler G, et al. (2006). FGF-4 signaling is involved in mir-206 expression in developing somites of chicken embryos. Dev. Dyn. 235: 2185-2191.

Sweetman D, Goljanek K, Rathjen T, Oustanina S, et al. (2008). Specific requirements of MRFs for the expression of muscle specific microRNAs, miR-1, miR-206 and miR-133. Dev. Biol. 321: 491-499. 
van Rooij E, Sutherland LB, Qi X, Richardson JA, et al. (2007). Control of stress-dependent cardiac growth and gene expression by a microRNA. Science 316: 575-579.

Visone R, Pallante P, Vecchione A, Cirombella R, et al. (2007). Specific microRNAs are downregulated in human thyroid anaplastic carcinomas. Oncogene 26: 7590-7595.

Wang R, Hu Y, Song G, Hao CJ, et al. (2012). MiR-206 regulates neural cells proliferation and apoptosis via Otx2. Cell Physiol. Biochem. 29: 381-390.

Zhao Y, Samal E and Srivastava D (2005). Serum response factor regulates a muscle-specific microRNA that targets Hand2 during cardiogenesis. Nature 436: 214-220.

Zuker M (2003). Mfold web server for nucleic acid folding and hybridization prediction. Nucleic Acids Res. 31: $3406-$ 3415. 